\title{
Entropy Applied to Morphological Analysis and Modelisation of Nanomaterial Optical Properties
}

\author{
Christine Andraud $\left({ }^{1}\right)$, Jacques Lafait $\left({ }^{1, *}\right)$, Azeddine Beghdadi $\left({ }^{2}\right)$ and \\ Joaquina Peiro $\left({ }^{1}\right)$ \\ $\left({ }^{1}\right)$ Laboratoire d'Optique des Solides de l'Université Pierre et Marie Curie ( ${ }^{*}$, Case 80, \\ 4 place Jussieu, 75252 Paris Cedex 05, France \\ $\left({ }^{2}\right)$ Laboratoire des Propriétés Mécaniques et Thermodynamiques des Matériaux, \\ Université Paris Nord, Institut Galilée, avenue J. B. Clément, 93430 Villetaneuse, France
}

(Received 17 June 1996, accepted 9 December 1996)

PACS.78.20.-e - Optical properties of bulk materials and thin films

\begin{abstract}
The normalized configuration entropy, based on the theory of information, when applied to the image of heterogeneous media, points out a characteristic length of the disorder, $\ell_{\mathrm{opt}}$, at which we calculate the optical properties. The models we propose make a partition of the image between percolated and non percolated cells of size $\ell_{\text {opt }}$, in which we calculate effective dielectric functions. Two models are then developed performing respectively a coherent and a non coherent treatment in the calculation of the optical properties of the whole medium. The coherent model gives a good account of the metallic grain resonance and of the infrared behavior of both reflectance and transmittance of granular gold films, close to the percolation threshold, domain where the effective medium theories fail.
\end{abstract}

Résumé. - L'entropie de configuration normalisée, basée sur la théorie de l'information et appliquée à l'image d'un milieu hétérogène, permet de mettre en évidence une longueur caractéristique du désordre, $\ell_{\mathrm{opt}}$, à laquelle nous calculons les propriétés optiques. Les modèles que nous proposons effectuent une partition de l'image entre cellules de taille $\ell_{\mathrm{opt}}$, percolées et non percolées, dans lesquelles nous calculons une fonction diélectrique effective. Deux modèles ont ainsi été développés, réalisant respectivement un traitement cohérent et incohérent lors du calcul des propriétés optiques du milieu global. Le modèle cohérent rend bien compte de la résonance de grains métalliques et du comportement infrarouge de la réflexion et de la transmission de films d'or granulaires, aux alentours du seuil de percolation, hors du domaine de validité des théories de milieu effectif.

\section{Introduction}

The morphology of a medium can be analyzed as a quantity of information, as defined by Shannon [1] and Brillouin [2]. This quantity is a characteristic of the disorder of an image, related to a given statistical state of configuration, and to an entropy. We have adapted these information and entropy concepts to the image analysis of random two phase media $[3,4]$.

$\left({ }^{*}\right)$ Author for correspondence

(*) Unité associée au CNRS D0781

(C) Les Éditions de Physique 1997 
This analysis leads to a length, characteristic of the image, at which we choose to calculate the optical properties of the material (Sect. 1). In Section 2, we propose two models of the optical properties, based on the same principle of the image partition [5]. The first model uses a self consistent calculation, while the second allows a local calculation of the optical properties. We finally compare the predictions of our models to the predictions of classical models (Bruggeman, Maxwell Garnett, renormalization) and to optical measurements on gold granular films prepared by thermal evaporation.

\section{Normalized Configuration Entropy}

1.1. Definition. - The binary image is composed of black pixels (gray level: 0), considered as active, and white pixels (gray level: 1 ). It is analyzed through a sliding window of variable size $\ell$. We define the relative frequency $p_{k}(\ell)$ of occurrence of a cell of size $\ell$ enclosing $k$ black pixels in the image as:

$$
p_{k}(\ell)=\frac{N_{k}(\ell)}{N(\ell)}
$$

where $N_{k}(\ell)$ is the number of cells of size $\ell$ containing $k$ black pixels, and $N(\ell)$ is the total number of cells of size $\ell$. The number of possible configurations is: $\ell^{2}+1$. The entropy is defined as the sum over $k$ of all information quantities of each event $k$ weighted with their own weight $p_{k}(\ell)$ :

$$
H(\ell)=\sum_{k=0}^{\ell^{2}} p_{k}(\ell) \ln \left[p_{k}(\ell)\right] .
$$

In order to compare the different entropies calculated for various sizes of the cell of analysis, we need to normalize the entropy, by dividing it by the theoretical maximum entropy $\left(H_{\max }^{\mathrm{Th}}\right)$. $H_{\max }^{\mathrm{Th}}$ is determined by using the Lagrange Multiplier Method, with the single constraint:

$$
\sum_{k=0}^{\ell^{2}} p_{k}(\ell)=1 .
$$

We obtain $H_{\max }^{\mathrm{Th}}=\ln \left(\ell^{2}+1\right)$, which is the entropy corresponding to the equally probable distribution $\left(p_{k}=1 /\left(\ell^{2}+1\right)\right.$ for all $k$ values). We thus define the normalized configuration entropy:

$$
H^{*}(\ell)=\frac{\sum_{k=0}^{\ell^{2}} p_{k}(\ell) \ln \left[p_{k}(\ell)\right]}{\ln \left(\ell^{2}+1\right)} .
$$

1.2. Application to a Simulated Image. - We applied this calculation to a simulated image obtained by random trials of disk of 11 pixels in diameter, allowed to penetrate. The image size is $L^{2}=512 \times 512$ pixels. The background of the initial image is white, the disks are black. The black pixel concentration (surface fraction) inside the image is $20 \%$ (Fig. 1). Figure 1 shows the variation of the normalized configuration entropy with the size of the cell of analysis. The entropy presents a maximum value $\left(H_{\max }^{*}\right)$ for a size that we will now call the optimum length $\ell_{\text {opt }}$.

The optimum length is the size of observation at which the image presents a maximum of disorder, at which a maximum of different configurations can be obtained, at which the probability distribution is the closest to the equally probable distribution. 


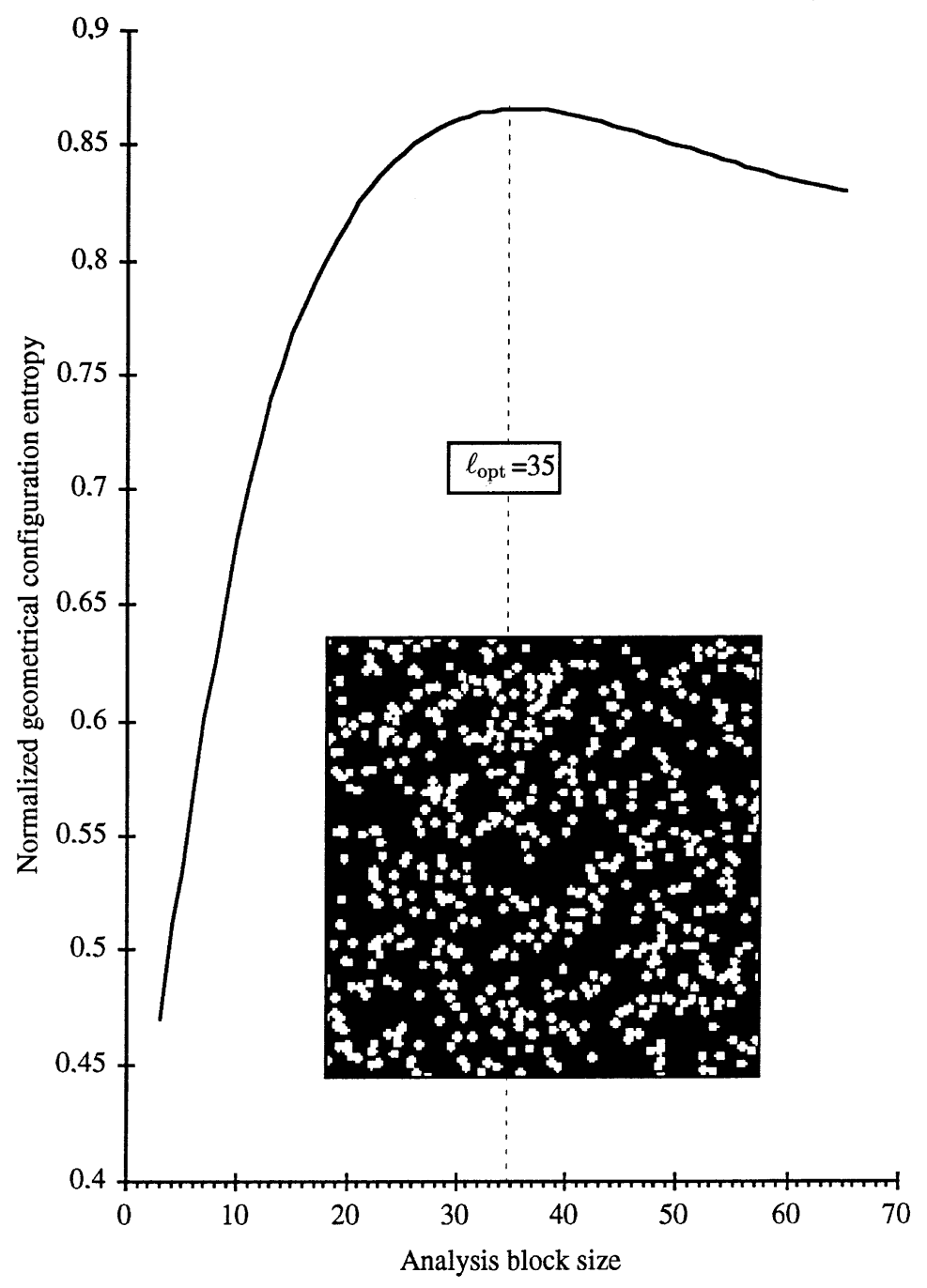

Fig. 1. - Normalized entropy of an image simulated by a random trial of penetrating disks (diameter 11 pixels, surface fraction $20 \%$ ) versus the size of the analysis cell.

1.3. Evolution of Entropic Parameters. - Let us now look at the variations of the optimum length and of the maximum value of the normalized configuration entropy versus the concentration in an image obtained by the same process as in Section 1.1. We generated a set of nine images of random trials of disks of 11 pixels in diameter, allowed to penetrate, with a black pixel concentration ranging from $10 \%$ to $90 \%$. The percolation threshold of this type of images is experimentally obtained at $60 \%$, with 4 neighbor labelling.

The two entropy parameters $\left(H(\ell)\right.$ and $\left.\ell_{\text {opt }}\right)$ present an extremum (Fig. 2) around the percolation: the maximum value of the normalized configuration entropy increases with the concentration until $53 \%$, and then decreases. The optimum length presents a plateau of minimum values centered at $60 \%$. This minimum value of $\ell_{\mathrm{opt}}$ is equal to 11 pixels, size of the structuring element of the image, diameter of the elementary disk. This length is the size of 


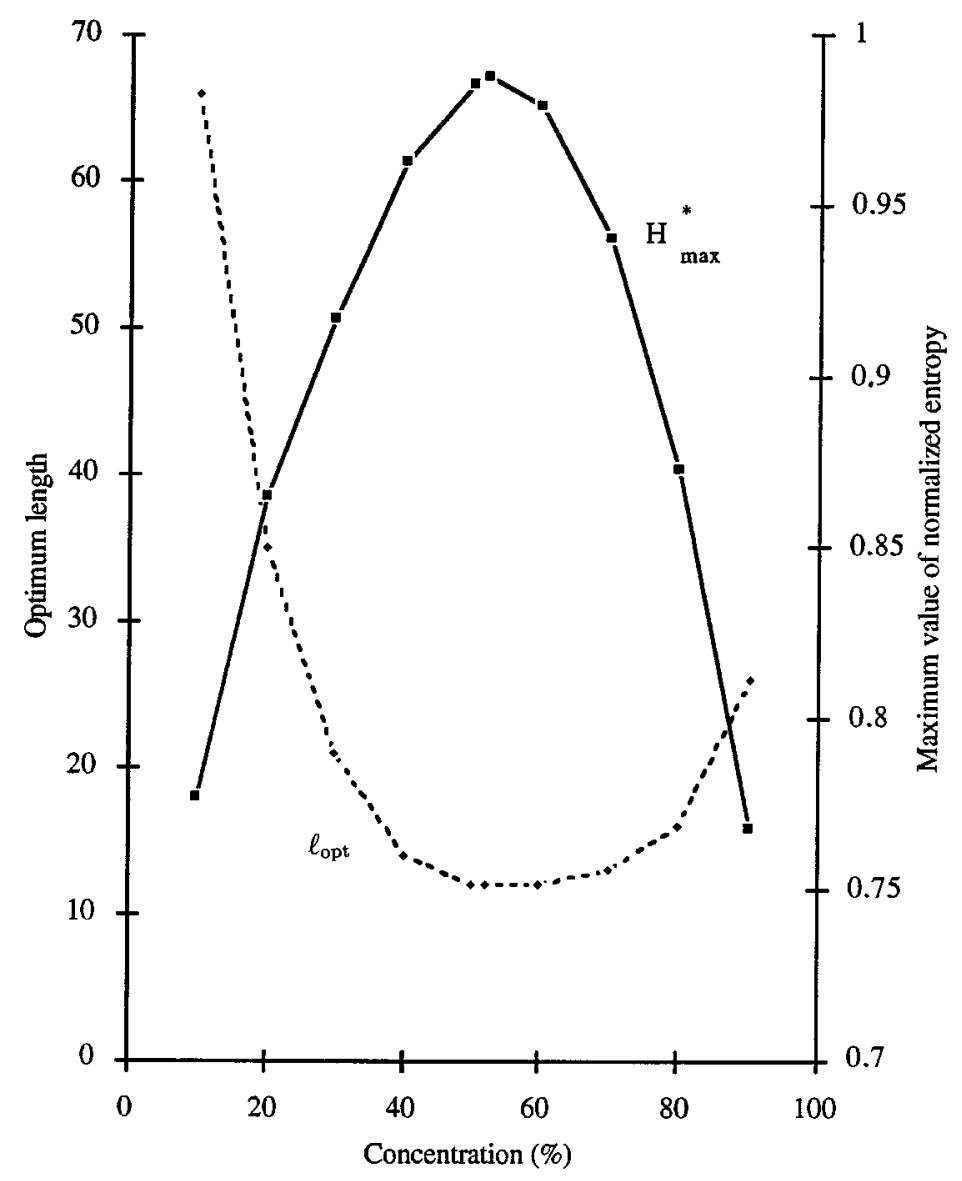

Fig. 2. - Variations of the entropic parameters of the images of random penetrating disks with the black pixel concentration.

the smallest element which, if added, make the medium pass from non percolating state to percolating state. It is the size at which we decide to calculate the optical properties, because associated with a maximum of realizable configurations, without any redundancy or lack of information. Each configuration having its own local optical response, will then affect the global response of the medium.

\section{Optical Models}

Our models are based on the model previously proposed by Hilfer $[5,6]$ for porous media. In our case, we are interested in accounting for the optical properties of heterogeneous media generally composed of metallic inclusions of nanometric size, embedded in a dielectric matrix. In the images of these media, white pixels correspond to the dielectric component (dielectric function: $\epsilon_{\mathrm{D}}$ ), and black pixels to the metallic component (dielectric function: $\epsilon_{\mathrm{M}}$ ). By contrast with the effective medium theories which make a partition of the whole image between the two 
components (metal and dielectric), our models:

(1) make a partition of the medium at size $\ell_{\text {opt }}$, by using a sliding window; giving $(L-\ell+1)^{2}$ cells;

(2) separate these cells in two classes: percolating and non percolating cells.

The state of percolation of each cell of concentration $\mu$ in black pixels is obtained by looking for a conducting path between two opposite edges of the cell, with 4 neighbor labelling. In both classes, we calculate the local effective dielectric function with the Maxwell Garnett model [7], modified by Cohen et al. [8]: a percolating cell is assimilated to an equivalent cell composed of a dielectric sphere embedded in a metallic matrix, with dielectric concentration $(1-\mu)$; a non percolating cell is assimilated to an equivalent cell composed of a metallic sphere embedded in a dielectric matrix, with the metal concentration $\mu$. One thus gets a series of effective dielectric functions $\epsilon_{i \mathrm{P}}$ and $\epsilon_{k \mathrm{NP}}$ respectively for a percolating $i$ cell and a non percolating $k$ cell:

$$
\begin{aligned}
\epsilon_{i \mathrm{P}} & =\epsilon_{\mathrm{M}} \frac{2 \epsilon_{\mathrm{M}}+\epsilon_{\mathrm{D}}+2\left(1-\mu_{i}\right)\left(\epsilon_{\mathrm{D}}-\epsilon_{\mathrm{M}}\right)}{2 \epsilon_{\mathrm{M}}+\epsilon_{\mathrm{D}}-\left(1-\mu_{i}\right)\left(\epsilon_{\mathrm{D}}-\epsilon_{\mathrm{M}}\right)} \\
\epsilon_{k \mathrm{NP}} & =\epsilon_{\mathrm{d}} \frac{2 \epsilon_{\mathrm{D}}+\epsilon_{\mathrm{M}}+2 \mu_{k}\left(\epsilon_{\mathrm{M}}-\epsilon_{\mathrm{D}}\right)}{2 \epsilon_{\mathrm{D}}+\epsilon_{\mathrm{M}}-\mu_{k}\left(\epsilon_{\mathrm{M}}-\epsilon_{\mathrm{D}}\right)} .
\end{aligned}
$$

In order to distinguish between two types of possible electromagnetic propagation in these heterogeneous media, we then developed two different models on this basis. In the first model, we make a self consistent calculation of the effective dielectric function $\epsilon_{\mathrm{e}}$ of the whole image by using a Bruggeman model [9] generalized to $(L-\ell+1)^{2}$ components. One writes that the polarizability of all these components embedded in the effective medium is equal to zero:

$$
\underbrace{\sum_{i} \lambda_{\mathrm{P}}^{i} \frac{\epsilon_{i \mathrm{P}}-\epsilon_{\mathrm{e}}}{\epsilon_{i \mathrm{P}}+2 \epsilon_{\mathrm{e}}}}_{\begin{array}{c}
\text { Sum on the } \\
\text { percolating blocks }
\end{array}}+\underbrace{\sum_{k} \lambda_{\mathrm{NP}}^{k} \frac{\epsilon_{k \mathrm{NP}}-\epsilon_{\mathrm{e}}}{\epsilon_{k \mathrm{NP}}+2 \epsilon_{\mathrm{e}}}}_{\begin{array}{c}
\text { Sum on the non } \\
\text { percolating blocks }
\end{array}}=0 .
$$

Where $\lambda_{\mathrm{P}}^{i}$ and $\lambda_{\mathrm{NP}}^{k}$ are respectively the proportions of percolating and non percolating cells with concentrations $\mu_{i}$ and $\mu_{k}$. We then calculate the optical properties (the reflectance $R$ and the transmittance $T$ ) of the medium, by using the Abelès formulae [10].

In the second model, we make a local calculation of the optical properties $\left(R_{i}, T_{i}\right)$, in each cell by using the local dielectric function (Eqs. (4) and (5)) and then applying the Abelès formulae. A simple summation on all cells will simply give the optical properties $(R, T)$ of the whole medium:

$$
(R, T)=\sum_{i}\left(R_{i}, T_{i}\right)
$$

In the first model, the application of the effective medium treatment up to the size of the whole medium is equivalent to a coherent treatment, with the same value of the phases of all the local electromagnetic fields. A calculation taking into account the exact phases of the fields is beyond the scope of this work. In the second model, we explore the effect of an incoherent treatment directly applied from the size $\ell_{\mathrm{opt}}$.

\section{Application to a Real Material}

We show now the application of the new morphological analysis tool, normalized configuration entropy, and of the two presented optical models, to the real image of a thin discontinuous gold film. 


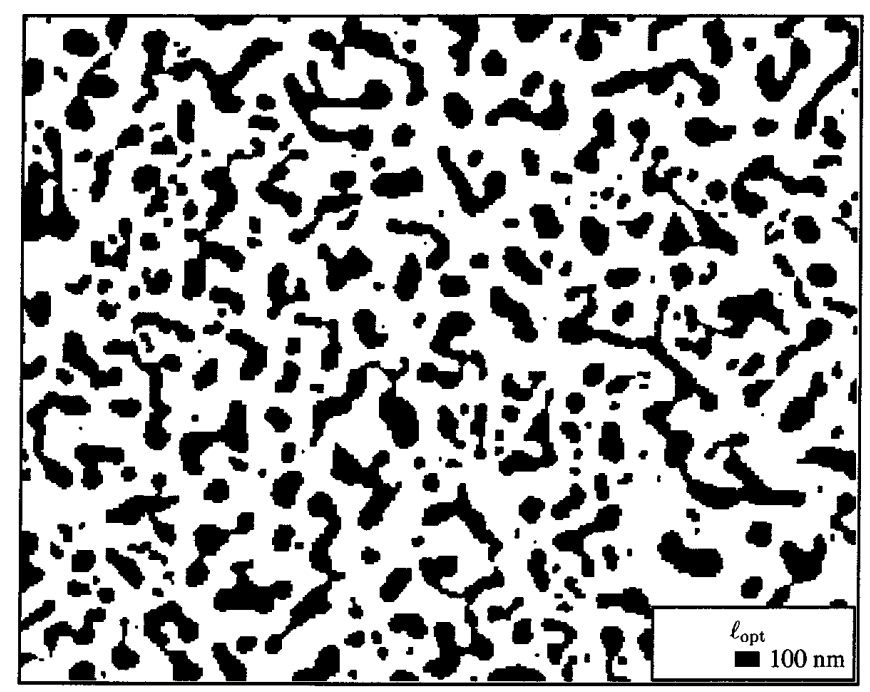

Fig. 3. - Image of a thin gold film, metal fraction $30 \%$.

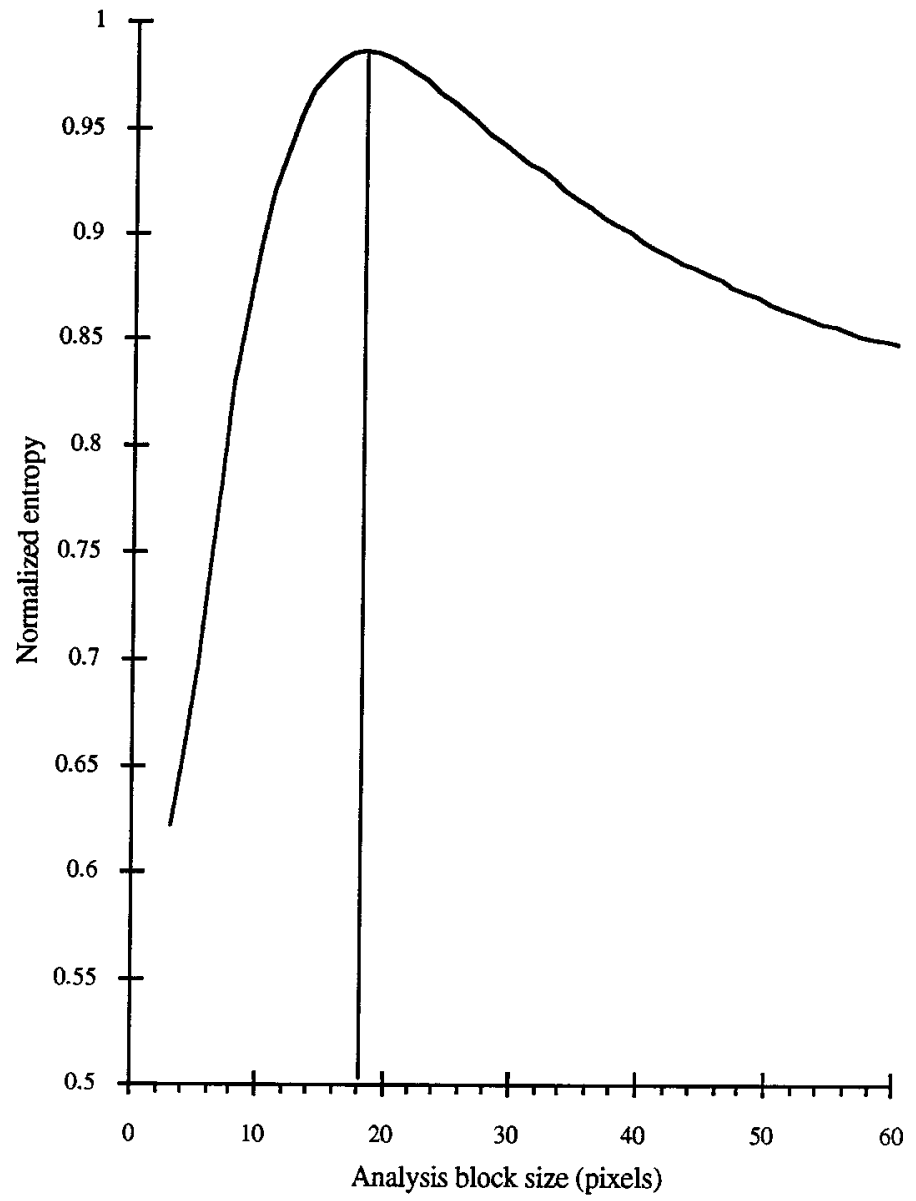

Fig. 4. - Normalized configuration entropy of the thin gold film (image in Fig. 3). 

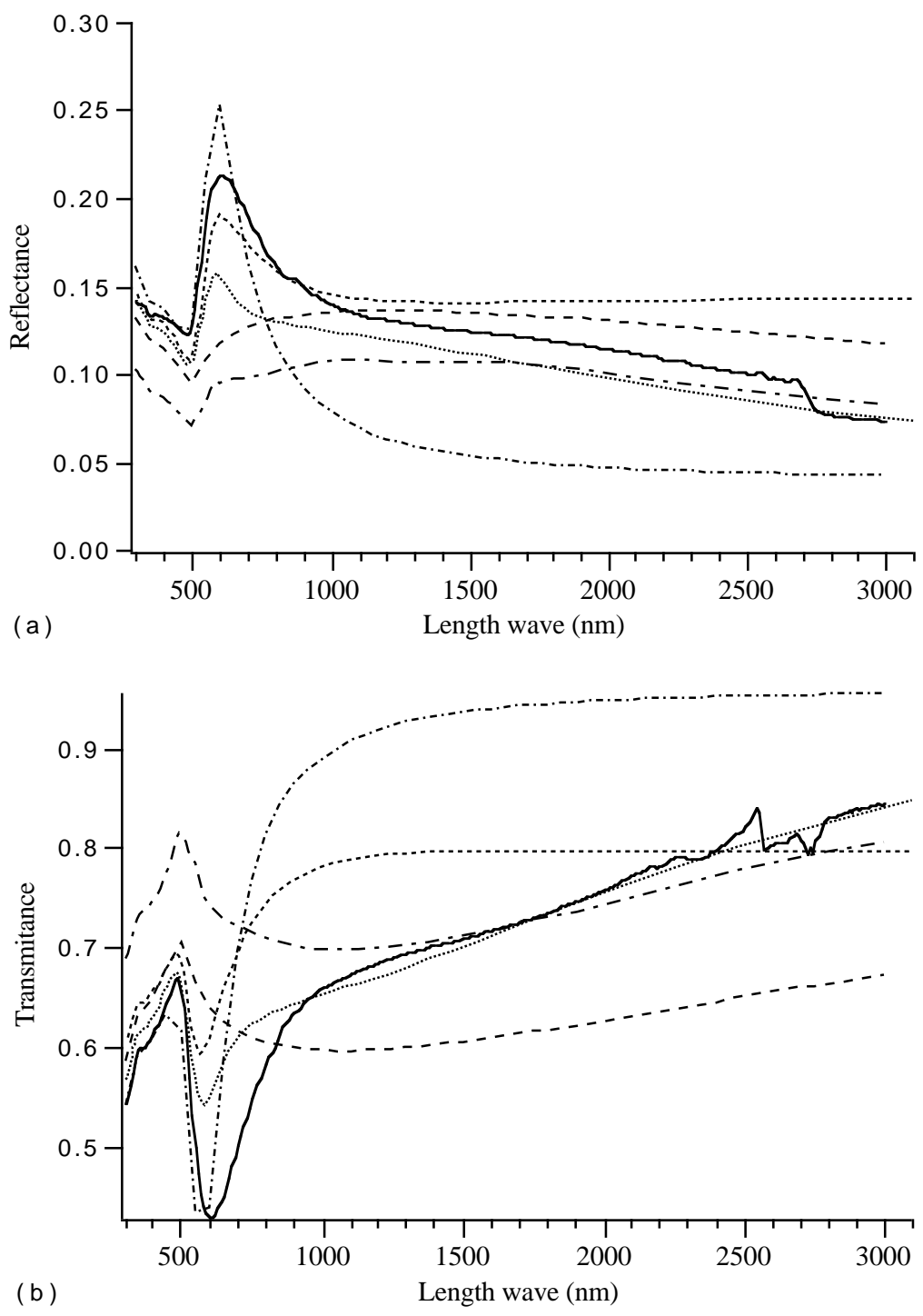

Fig. 5. - Reflectance (a) and Transmittance (b) of the thin gold film (image in Fig. 3): (-) Measurement; (...) Model 1; (- - -) Model 2 (-.-.-) Renormalization; (-.-) Maxwell Garnett; (- -) Bruggeman.

The deposition of the film is realized by thermal evaporation under ultra high vacuum. Its mass thickness is equal to $d=61 \AA$. The image was binarized, giving a black pixel concentration $p=32 \%$ (Fig. 3). From these values, we deduce the "real" thickness of the film, $d / p=190 \AA$.

The entropic analysis gives an optimum length equal to 18 pixels, corresponding to $100 \mathrm{~nm}$ on the sample (Fig. 4). We compare the predictions of our models to the predictions of classical models (Bruggeman, Maxwell Garnett, renormalization [11]) and to optical measurements on the discontinuous film (Fig. 5).

The image and the experimental optical properties both point out the fact that the film is below the percolation threshold. The decrease of the reflectance $R$ and the increase of the 
transmittance $T$ with wavelength are indeed characteristic of a dielectric behavior of the whole medium.

The Maxwell Garnett theory gives a relatively good account of the position and amplitude of the metallic grain resonance observed around $\lambda=600 \mathrm{~nm}$, but does not fit correctly the optical properties in the near infrared.

On the opposite, the Bruggeman model accounts well for the optical properties in the near infrared, but is unable to reproduce the grain resonance. This result confirms that the Bruggeman model predicts a too broad and dumped grain resonance.

The renormalization theory gives also satisfactory agreements in the near infrared, but underestimates the grain resonance. It can be due to the fact this model does not use directly the metal concentration, which is the main parameter determining the position and amplitude of this resonance.

Our first model accounts well for the properties in the near infrared, and gives the best prediction for the grain resonance, although its amplitude is weaker and its width is narrower than the experimental resonance. This result confirms that this model takes better into account the geometry of the medium, the average concentration and the concentration distribution, which notably govern the resonance.

Our second model is not so efficient as the first one in the near infrared, but it is somewhat better for the reflectance near the resonance. Its relative failure as compared to the first model should come from the non coherent calculation performed from a too small size.

\section{Conclusion}

The entropic analysis and its associated models appear as good tools for the modelization of the optical properties of two phase heterogeneous media. A more systematic application of these tools to real and simulated media is in progress.

\section{Acknowledgments}

We wish to thank P. Gadenne for his help in the gold film deposition, and R. Hilfer for his theoretical collaboration.

\section{References}

[1] Shannon C., A mathematical theory of communication, Bell. Syst. Tech. J. 27 (1948) 623-656.

[2] Brillouin L., Science and information theory (Academic Press, New York, 1956).

[3] Beghdadi A., Andraud C., Lafait J., Peiro J. and Perreau M., Entropic and multifractal analysis of disordered morphologies, Fractals 1 (1993) 671-679.

[4] Andraud C., Beghdadi A. and Lafait J., Entropic analysis of random morphologies, Physica A 207 (1994) 208-212.

[5] Hilfer R., Geometric and dielectric characterization of porous media, Phys. Rev. B 44 (1991) 60-75.

[6] Boger F., Feder J., Jossang T. and Hilfer R., Microstructural sensitivity of local porosity distributions, Physica A 187 (1992) 55-70. 
[7] Maxwell Garnett J.C., Colours in metal glasses and in metallic films, Philos. Trans. R. Soc. Lond. 203 (1904) 385-420.

[8] Cohen R.W., Cody G.D., Coutts M.D. and Abeles B., Optical properties of granular silver and gold films, Phys. Rev. B 8 (1973) 3689-3701.

[9] Bruggeman D.A.G., Berechnung verschiedener physikalischer Konstanten von heterogenen Substanzen, Ann. Phys. (Leipz.) 24 (1935) 636-664.

[10] Abeles F., Optics of thin films, in Advanced optical techniques (A.C.S. Van Heel, Ed., North Holland, Amsterdam, 1967) pp. 143-188.

[11] Berthier S., Driss Khodja K. and Lafait J., Optical dielectric function of inhomogeneous media calculated by the Kadanoff's block method, Europhys. Lett. 4 (1987) 1415-1420. 